\title{
The role of the mesoscale in migration kinetics of flat grain boundaries
}

\author{
C P Race* J von Pezold, and J Neugebauer \\ Max Planck Institute for Iron Research, 40237 Dusseldorf, Germany
}

\begin{abstract}
Classical molecular dynamics simulations of bicrystalline systems are a commonly used tool for exploring the migration of grain boundaries. Most simulation work to date has focused on measuring the mobility of grain boundaries, assuming it to be an intrinsic property of a boundary of a given geometry.

Here we present results from simulations of the migration of a typical high-angle grain boundary that show that the concept of intrinsic mobility fails for defect-free, flat boundaries of the type frequently simulated and that key assumptions often made in analysing the kinetics of migration do not hold. Our dynamical simulations of grain boundary migration show that the grain boundary velocity is not simply proportional to the driving force for grain boundary motion, as commonly assumed, and shows a strong and complex dependence on the system size.

By analysing the migration mechanism at the larger mesoscale we show that defect-free, flat boundaries must migrate via the homogeneous nucleation and growth of islands of transformed crystal volume on the grain boundary surface. We present a detailed analysis of the kinetics of this process, which only emerges in simulations of large grain boundary areas. An island-based mesoscale mechanism implies an energy barrier for migration that is inversely proportional to the driving force for migration - in the experimental (zero-force) limit such boundaries must be immobile. This calls into question the concept of an intrinsic mobility for defect-free, flat grain boundaries and suggests that mobility of real boundaries at low temperatures is rather a function of their morphology and defect content and at high temperatures is a result of thermal roughening.
\end{abstract}

\section{INTRODUCTION}

The pattern of grain boundaries in a polycrytalline material, embodied in the texture, grain size distribution and grain misorientation distribution, is a major determinant of that material's properties. The migration of grain boundaries thus plays a major role in the evolution of mechanical properties during recrystallization and mechanical processing and in application. A detailed understanding of the process of grain boundary migration is thus of great value.

Extensive experimental studies of bicrystals and polycrystals over the last sixty years [1] have established a detailed picture of the variation of grain boundary mobility with the five macroscopic degrees of freedom of grain boundary structure [2, 3], with boundary shape [4], with temperature and driving force [5, 6] and with impurity content 7]. More recently, computational studies have also played a role [8 21]. Simulations of grain boundary motion are easier to control than experiments and yield detailed information about the migration process down to the atomistic length-scale. However, constraints on computational expense in classical molecular dynamics (MD) mean that simulations are restricted to relatively small system sizes (typically $10^{4}-10^{6}$ atoms) and must use unrealistically high driving forces (typically between 10 and $250 \mathrm{MPa}$, compared with realistic forces between $10^{-4}$ and $\left.1 \mathrm{MPa}[22,23]\right)$ to achieve grain boundary migration on accessible time-scales.

\footnotetext{
* Contact: christopher.race@manchester.ac.uk Current address: Dalton Nuclear Institute, University of Manchester, Manchester M13 9PL, United Kingdom
}

A key grain boundary property measured in migration simulations is the mobility, $m$, defined as the rate of change of the steady state grain boundary velocity, $v$, with driving force, $P$, in the low force limit, $m=\mathrm{d} v /\left.\mathrm{d} P\right|_{P=0}$ [22]. This mobility is assumed to be an intrinsic property of a given grain boundary geometry (i.e. for a given point in the five-dimensional configuration space of the macroscopic degrees of freedom of grain boundary geometry [23]). In order to study the grain boundary mobility for a fixed geometry, flat, as opposed to curved, boundaries are commonly simulated. To extract the mobility from a simulated grain boundary trajectory, a simple barrier hopping model of the migration kinetics is used. This model is frequently further simplified by making certain assumptions, which we will discuss in detail in Section $\amalg$ A.

Previous simulations have largely focused on relatively small simulation cells with grain boundary areas $\sim 10^{3} \AA^{2}$ (containing $\sim 10^{3}$ grain boundary atoms). These simulations have shed much light on the variation of the grain boundary mobility and activation energy for migration as a function of grain boundary geometry [13, 19]. They have also permitted detailed studies of the atomistic migration mechanisms of selected boundaries [18, 20].

The mechanism of boundary migration on larger length-scales has not been studied in detail. At the mesoscale, which we define here as a length-scale on which the grain boundary can be regarded as a twodimensional dividing surface between regions of crystal with different orientations, a plausible mechanism, proposed by Mott [24] (and developed further by Smoluchowski [25]), involves the formation of islands of migrated crystal volume. No definitive evidence for this 
process has previously been presented, although several results from experiment [26, 27] and simulation [11, 28] support the suggestion. Simulations of shear-coupled migration have been used to study a related process involving the nucleation of pairs of disconnections [29].

In this paper, we present the results of classical MD simulations of the migration of a [111] $\Sigma 7$ symmetric tilt boundary, a boundary much studied in previous simulations and experiments $7,[10,13$, 16, 21]. We have focused on a flat grain boundary of the type often used to determine intrinsic mobilities in simulations and in experiments. In particular, we have analysed the behaviour of a defect-free (smooth) flat boundary, i.e. a boundary that is below its roughening transition temperature [12] and that contains no structural defects (such as secondary grain boundary dislocations) inherent to its relaxed configuration. Based on these results we show that a process of homogeneous nucleation and growth determines the kinetics of grain boundary migration, but that this behaviour only emerges when large super-cells are used. We further show that the nucleation process implies that the energy barrier for migration increases rapidly as the driving force decreases; in the experimental limit of low driving force smooth boundaries are strictly immobile. Crucially, we find that the size of the simulation cell has a strong effect on the grain boundary velocity and can change the nature of the migration mechanism. To guarantee converged behaviour we require unexpectedly large three-dimensional simulation cells.

In Section $\llbracket A$ we briefly review the commonly employed barrier model of migration kinetics. We question some of the simplifying assumptions that are often made and show how different assumptions lead to an alternative approach to deriving grain boundary kinetic parameters, which is perhaps more generally applicable. In Section IB we briefly outline the computational approach that we have used.

Section 【II presents our results. In Section ЩIA we show that the migration velocities measured in our simulations violate the commonly made assumptions about migration kinetics. Section 【IIB considers the migration mechanism at the mesoscale. We show that defect-free, flat grain boundaries of the type that we have simulated move via the nucleation and growth of islands of transformed crystal volume on the grain boundary surface. The rate determining step for boundary migration is thus the formation of a stable island nucleus - a process that entails a migration barrier inversely dependent on the driving force. We further show in detail how an analytical model of island-based migration kinetics can account quantitatively for the unexpected results presented in Section $\amalg A$.

In Section IV we consider the implication of our findings for future studies of grain boundary migration.

\section{METHODS}

\section{A. A model of migration kinetics}

A commonly used model of the kinetics of grain boundary migration considers a migration event as a thermally activated reaction, at temperature $T$, over an energy barrier of height $E_{\mathrm{A}}$ between two states representing the grain boundary in stable positions a distance $d$ apart 22]. This model is illustrated schematically in Figure 1, in which we also mark the energy of the grain boundary in its stable state $E_{0}$ and the energy in the activated state in the barrier configuration $E_{1}$, such that $E_{\mathrm{A}}=E_{1}-E_{0}$. With no driving force, in this simple model, the grain boundary will execute a one-dimensional random walk of forward and backward hops.

The application of a thermodynamic driving force for grain boundary migration can be incorporated as an energy penalty $\varepsilon$ applied to one of the two stable states such that its energy is raised to $E_{0}^{*}$. In general, this would also alter (and likely increase) the energy of the activated state, say to $E_{1}^{*}$. We define $e=E_{1}^{*}-E_{1}$ as the shift in the energy of the activated state. The progress of the grain boundary is now a biased competition between forward and backward hops of length $d$, implying a velocity of

$$
\begin{aligned}
v(\varepsilon, T)=f(\varepsilon) d[\exp (- & \left.\left(E_{\mathrm{A}}-\varepsilon+e\right) / k_{\mathrm{B}} T\right) \\
- & \left.\exp \left(-\left(E_{\mathrm{A}}+e\right) / k_{\mathrm{B}} T\right)\right] \\
=f(\varepsilon) d \exp \left(-E_{\mathrm{A}} / k_{\mathrm{B}} T\right) & \exp \left(-e / k_{\mathrm{B}} T\right) \\
\times[ & \left.\exp \left(\varepsilon / k_{\mathrm{B}} T\right)-1\right],
\end{aligned}
$$

where the frequency $f(\varepsilon)=f_{0} \exp \left(S_{\mathrm{A}}(\varepsilon) / k_{\mathrm{B}}\right)$ is a function of the driving force $\varepsilon$ and depends on an "attempt frequency" $f_{0}$ and the activation entropy $S_{\mathrm{A}} 22$.

At this stage in most analyses it is implicitly assumed that the application of a driving force does not affect the height of the barrier for forward hops, but makes reverse hops less likely, i.e. $e=\varepsilon[30]$. It is also usual to assume that $\varepsilon \ll k_{\mathrm{B}} T$, so that $\exp \left(\varepsilon / k_{\mathrm{B}} T\right) \approx 1+\varepsilon / k_{\mathrm{B}} T$ and the velocity becomes

$$
v(\varepsilon, T) \approx f(\varepsilon) d\left[1 / k_{\mathrm{B}} T\right] \exp \left(-E_{\mathrm{A}} / k_{\mathrm{B}} T\right) \varepsilon,
$$

linear in the driving force [31]. This then leads to the definition of an intrinsic mobility $m(T)$ for a given boundary,

$$
m(T) \equiv \lim _{\varepsilon \rightarrow 0} v(\varepsilon, T) / \varepsilon .
$$

The two assumptions used to obtain Eq. (2) require that we are in the low driving force limit $\varepsilon \ll E_{\mathrm{A}}$, and $\varepsilon \ll k_{\mathrm{B}} T$. Whilst these assumptions are reasonable for migrating grain boundaries in real materials, MD simulations employ very large driving forces. We must also be 
careful about the meaning of the energies in the above discussion: they refer not to a single atom, but to the unit of our system taking part in the rate determining step, which will involve multiple, say $n$, atoms, where $n$ might be quite large. Writing our driving force either as an excess energy $\epsilon$ per atom or a pressure (energy per unit volume) $P$ then gives us $\varepsilon=n \epsilon=n P \Omega$, where $\Omega$ is the volume per atom. $n$ may well be $\sim 10-100$ (we do not know, a priori) and $\epsilon \sim 0.01 \mathrm{eV} /$ atom which gives an energy shift $\varepsilon \sim 0.1-1 \mathrm{eV}$. This is exactly in the range of activation energies $E_{\mathrm{A}}$ measured in many simulations and similar to typical values of $k_{\mathrm{B}} T \sim 0.05-0.1 \mathrm{eV}$.

The driving force in typical simulations is thus quite high and the linear relation in Eq. (2) cannot be relied upon. However, alternative assumptions may be made in order to simplify Eq. (10). Whilst the motion of a grain boundary can be considered as a one-dimensional random walk, typical trajectories (from our own simulations and from the literature) tend to show grain boundaries hopping in the forward direction only, at a rate dependent on the driving force. Reverse jumps are effectively frozen out because $\left(E_{\mathrm{A}}+e\right) \gg k_{\mathrm{B}} T$ (remembering that $e$ depends on $\varepsilon)$. Making the assumption of a high driving force limit $\varepsilon \gg k_{\mathrm{B}} T$ allows us to write $\exp \left(\varepsilon / k_{\mathrm{B}} T\right)-1 \approx \exp \left(\varepsilon / k_{\mathrm{B}} T\right)$ and so

$$
v(\varepsilon, T)=f(\varepsilon) d \exp \left[-E_{\mathrm{A}}^{*}(\varepsilon) / k_{\mathrm{B}} T\right],
$$

where $E_{\mathrm{A}}^{*}(\varepsilon) \equiv E_{\mathrm{A}}+e-\varepsilon$ is the effective energy barrier for a one way hopping process. We will make use of this expression in analysing the results of our simulations in Section 【II

\section{B. Migration simulations}

We have carried out a series of classical MD simulations of a pair of moving grain boundaries across a range of driving forces and temperatures using the Lammps software package [32]. Our chosen grain boundary is the [111] $\Sigma 7$ symmetric tilt boundary with $\{321\}$ boundary planes. We have selected this boundary because it is typical of low-period high-angle CSL boundaries and because it has been observed to be highly mobile in previous work [13], so that the computational expense of our detailed studies is reduced.

We used simulation cells of between 1500 and 1.2 million atoms (giving grain boundary areas of between $13 \times 14$ and $425 \times 340 \AA^{2}$ ) containing a pair of grain boundaries and with periodic boundary conditions in all three directions. The cell size was held constant in the two directions parallel to the grain boundary plane and a zero-pressure Nosé-Hoover barostat was applied to the third direction (parallel to the direction of motion of the grain boundaries). These boundary conditions were a compromise: we needed to hold two dimensions fixed because MD simulations always contain an unrealistic area of grain boundary per unit volume and so the surface tension in the grain boundary would otherwise have tended to distort the cell [33]. The third dimension was allowed to relax because the driving force that we used was such that an excess pressure would otherwise have built up as the boundary migrated, changing the effective driving force over time. We applied a Nosé-Hoover thermostat to the system, since the conversion of crystal volume from the disfavoured to the favoured orientation released a significant amount of energy, which would otherwise have raised the temperature and progressively altered the grain boundary mobility.

Our use of periodic boundary conditions in the direction normal to the grain boundary plane will prohibit any shear-coupled grain boundary migration of the type proposed for high angle boundaries by Cahn et al. 34] and extensively studied in the theoretical 35, 36], computational [29, 37, 38] and experimental [6, 39, 40] literature. However, simulations that we have carried out in cells containing a single boundary and with free ends reveal that shear-coupled migration of our chosen boundary does not spontaneously occur (though it might be enforced if an appropriate shear stress were applied to the ends of the simulation cell) and so must involve a mechanism (probably also nucleation mediated [40]) with a significantly higher energy barrier than those we explore here.

The inter-atomic forces in our simulations were calculated using an embedded atom model (EAM) potential fitted to properties of aluminium derived from experiment and density functional theory calculations [41]. The migration of our grain boundaries was driven using an artificial force derived from a potential that added an energy penalty to each atom based on the positions of its nearest neighbours [10]. This form of driving force (along with other similar forces 14, 21]) has been used extensively in previous studies [8 10, 13, 14, 21]. It offers the advantage of being equally easy to apply to any boundary geometry.

We have analysed the forces on individual atoms and found that at the temperatures we study the artificial driving force makes a contribution of only a few percent to the typical force on an atom due to thermal fluctuations; it is thus acting correctly as a thermodynamic bias to the underlying motion of the grain boundary (i.e. it should not significantly impact the paths taken by atoms during the migration events).

We initialised our simulation cells with atomic velocities distributed according to our chosen temperature and then allowed at least 20 ps for equilibration before switching on the driving force. We then ran the simulations for up to a further $5 \mathrm{~ns}$ (with a time-step of $1 \mathrm{fs}$ ) to allow the boundaries to traverse the length of the cell (each boundary moved up to $100 \AA$ ). We determined the position of the grain boundary, on average and as a function of position across the grain boundary surface, by examining the atomic order parameter used to calculate the artificial driving potential. We then calculated the average velocity from the position-time data with an estimate of the error given by bootstrap resampling of the 
trajectory [42]. This approach exploits the fact that even a single trajectory consists of multiple migration events and so contains information about the random nature of grain boundary migration. A sufficiently long trajectory can therefore yield the same statistical data as multiple shorter simulations if treated correctly. Due to thermal fluctuations in the favoured grain, the true driving force was slightly reduced from the nominal value in the artificial potential. We calculated the necessary adjustments via thermodynamic integration as in Reference [13].

\section{RESULTS AND DISCUSSION}

\section{A. Kinetic properties from migration simulations}

Most analyses of grain boundary mobility, in both simulation and experiment, proceed by calculating a temperature-dependent mobility from an assumed proportionality between velocity and driving force (see Equation 3). The behaviour of $m(T)$ with $T$, assumed to be Arrhenius-like, is then used to calculate an activation energy for the boundary migration. Critically, in the case of simulations, the velocity data are typically derived using super-cells of a fixed and quite small size. Here we briefly present an analysis from small cell simulations of this sort for our $\Sigma 7$ boundary. We do this to highlight some immediate issues with this approach before presenting size-converged data from much larger systems.

Figure 2 presents the results of a series of grain boundary migration simulations across a broad range of temperatures $(400-800 \mathrm{~K})$ and driving forces in a fixed system size (containing 24 thousand atoms and with a grain boundary area of $53 \times 57 \AA^{2}$, with $\sim 1000$ boundary atoms). Typical studies in the literature use forces up to $\epsilon=25 \mathrm{meV} /$ atom $(P \sim 240 \mathrm{MPa})$, but we have also simulated migration at even more unrealistic forces (up to $\epsilon=100 \mathrm{meV} /$ atom, $P \sim 1 \mathrm{GPa}$ ) in order to explore impact of the driving force on the behaviour of the system. Thermal disorder in the positions of atoms reduces the free energy difference between the favoured and disfavoured grains below the nominal value of the applied artificial driving force for grain boundary migration. Adjustments to take into account this effect are indicated in Fig. 2. We immediately see from the behaviour of our sample boundary that the commonly assumed $v \propto \epsilon$ relationship does not hold in general. The inset to Fig. 2 shows the nominal mobility, defined as $v / \epsilon$, as a function of driving force. This is normally assumed to be a constant, but it clearly is not. At lower temperatures there appears to be a minimum threshold driving force, below which no motion of the grain boundary is observed. At a high temperature, around $700 \mathrm{~K}$ here, the behaviour is closer to that expected. At higher temperatures still, the velocity remains approximately proportional to the driving force, but the mobility decreases with increasing temperature. At all temperatures the asymptotic limit at high driving forces is a constant nominal mobility $(v / \varepsilon)$.

Non-linear behaviour is also presented and discussed by Deng and Schuh [9] though, as we discuss below, the origin of the behaviour in our case differs significantly from that in Ref. [9].

The complex results derived here are also consistent with recent experimental findings by Kang et al. [43], who have reported a nonlinear relationship between interface mobility and driving force and a threshold driving force for migration in experiments on $\mathrm{BaTiO}_{3}$.

For the above results an intrinsic mobility $m(T)$ is clearly ill defined, at least below $700 \mathrm{~K}$. This means that we cannot follow the usual procedure of determining an energy barrier for migration $E_{\mathrm{A}}$ from an Arrhenius relationship,

$$
m(T)=m_{0} \exp \left(-E_{\mathrm{A}} / k_{\mathrm{B}} T\right)
$$

To analyse our data, instead of the usual linear relation, Eq. (2), we will adopt the high driving force limit in Eq. (4). Taking the logarithm, we thus have,

$$
\ln (v)=\ln (f)-\left(\frac{E_{\mathrm{A}}^{*}}{k_{\mathrm{B}}}\right) \frac{1}{T},
$$

and we can use an Arrhenius plot of $\ln v$ against 1/T for each value of the driving force [44] $\epsilon$ to obtain the effective energy barrier $E_{\mathrm{A}}^{*}=E_{\mathrm{A}}+e-\varepsilon$, and prefactor $f$ as a function of $\epsilon$. A strong decrease in energy barrier for migration with increasing driving force can be seen in Figure 3. from which we can also see that a clear compensation effect [22, 45], a proportionality between the fitted values of $\ln f$ and $E_{\mathrm{A}}^{*}$, emerges. Such a compensation effect has often been reported in the values of $\ln f$ and $E_{\mathrm{A}}^{*}$ as grain boundary geometry (e.g. misorientation angle) is varied [5] and might be explained by variations in the geometrically necessary number of atoms involved in the migration process. However, it is not immediately clear why such a compensation effect should exist for a single boundary as the driving force strength is varied.

Molecular dynamics simulations of grain boundary migration are often carried out in small supercells, sometimes as small as $20 \times 20 \AA^{2}$ and 8000 atoms [13]. This means that the measured velocities could be influenced by finite system size effects. To investigate this issue we have carried out a series of migration simulations at $600 \mathrm{~K}$ in simulation cells across a wide range of sizes (between 1500 and 1.2 million atoms with grain boundary areas of between $13 \times 14$ and $425 \times 340 \AA^{2}$ ) and at several driving forces. As can be seen from Figure 4 the impact of system size is significant and takes a complicated form (which we will explain in Section IIIB).

Overall, the results presented above illustrate several important features of grain boundary migration, all of which require explanation. They are:

1. At lower temperatures the relationship of grain boundary velocity to the driving force is strongly 
non-linear, i.e. it does not follow the normally assumed relationship in Eq. (2).

2. At high temperature a linear relationship appears.

3. The velocity increases with temperature up to $700 \mathrm{~K}$ (which is the roughening temperature for our boundary), after which it begins to decrease.

4. At very high driving forces a constant nominal mobility $(v / \varepsilon)$ emerges.

5. The energy barrier for grain boundary migration decreases strongly with increasing driving force. This relationship is non-linear.

6. The kinetic pre-factor $f$ in Arrenhius fits to the migration data shows a compensation effect with varying driving force.

7. The measured grain boundary migration velocity varies with system size in a complex manner, which in turn is dependent on the driving force.

8. The velocity of the moving grain boundary does not converge to a stable value until the system size is very large compared with typical simulations in the literature.

In the following sections we will show that all of the above behaviour can be understood by analysing the mechanisms of grain boundary migration at the mesoscale.

\section{B. Mesoscale migration mechanism}

Several previous studies have investigated in detail the migration mechanisms of example grain boundaries at the atomistic scale, in terms of the pattern and timing of atomic rearrangements [20, 46]. We have undertaken similar studies for our $\Sigma 7$ boundary and find that at the atomistic level the boundary moves by the ordered shuffling of groups of 21 atoms (three times the unit of the CSL). These results will be presented in detail elsewhere. Much less information is available in the literature about how migration takes place on a larger mesoscale, on which the grain boundary can be regarded as a dividing surface between crystal grains with different orientations. It is the behaviour of the boundary at this larger length-scale that explains the complex sizedependent behaviour in our results. Various studies [12] have shown that, particularly at low temperature and driving force, grain boundaries commonly move rapidly between positions of particular stability, with periods of relative immobility between such jumps. The separation between the stable positions is determined by the grain boundary geometry and is typically of the order of several angstrom.

By studying the evolving shape of the grain boundary surface as it migrates in our simulations, we have observed that the mesoscale migration process is one whereby islands of crystal volume in the favoured orientation form at the grain boundary surface. These islands grow until they coalesce to give grain boundary motion. This island-based mechanism can be seen in the data shown in Figure 5. In the larger system we can clearly see that migration takes place via the formation and growth of four islands of migrated material. This type of boundary propagation is well known in surface epitaxy as layer by layer growth [47], but has, to our knowledge, not been reported in the context of grain boundary migration simulations.

The island-based mesoscale mechanism, which must be accommodated in order to obtain size-converged migration velocities, is illustrated schematically in Figure 6 . The rate limiting step is a homogeneous nucleation event and we will now present an analytical model of this mechanism. Consider an island of radius $r$ forming on a flat grain boundary surface. The formation of this island is penalised by the excess free energy density, $\phi(T)=\gamma-T \sigma$, associated with the island edge, where $\gamma$ and $\sigma$ are the internal energy and entropy per unit length of the island edge. When a driving force, $P=\epsilon / \Omega$, is present the island volume will be stabilised so that the excess free energy of the island can be written,

$$
F_{\text {island }}(r, P, T)=2 \pi r \phi(T)-\pi r^{2} P d,
$$

where $d$ is the edge height (the distance between stable grain boundary positions). We have calculated the energies of relaxed circular island structures in a cell of $265 \times 283 \times 77 \AA$ (336,000 atoms) using the identical EAM potential as for the dynamic simulations. The points in Figure 7 show the results. By fitting a straight line to the energies relaxed in the absence of any driving force (which correspond to $T=0 \mathrm{~K}$ ) we can obtain the value of the edge energy density for our system: we find $\gamma=31.5 \mathrm{meV}^{-1}$. Lines corresponding to the model in Eq. 7 with this value of $\gamma$ are shown in Fig. 7 and match the results of the molecular statics calculations well.

The model in Eq. 7 neglects any entropic effects in the volume (second) term. As discussed in Section $\amalg$ II at elevated temperatures the true value of the driving force (a free energy difference) is reduced from its nominal value, but this effect is small compared with the effect of the edge entropy density $\sigma$. We have also assumed that $\gamma$ has a constant value, whereas at very small values of the island radius the atomistic nature of the islands would affect their excess free energy. We can also see that for the largest driving force presented in Fig. 7 the model begins to deviate from the results of the molecular statics calculations. This is a consequence of the high driving force causing the island to expand slightly from its original, as constructed, size meaning that its excess energy is somewhat reduced.

Figure 7 reveals the key features of the island-based migration mechanism. At each driving force there is a critical radius, $r_{\text {crit }}(P, T)$, beyond which further growth of the island is thermodynamically favoured. The free 
energy of an island with this radius then corresponds to the free energy barrier for the rate limiting step in grain boundary migration: $F_{\mathrm{A}}(P, T) \equiv F\left(r_{\text {crit }}, P, T\right)$. As the driving force for migration increases, both the size and the energy of the critically stable islands decrease. This effect is confirmed in Figure 8, in which snapshots of migrating boundaries in dynamical simulations are shown. At a fixed system size increasing the driving force implies migration via a denser population of smaller islands.

Since the island-based mesoscale migration mechanism, rather than the local atomistic shuffle mechanism, determines the migration barrier it has important implications for the study of grain boundary migration with molecular dynamics simulations. A migration mechanism that is based on homogeneous nucleation implies that the migration barrier, and thus the mobility, will depend on driving force. This finding is in direct contrast to the commonly employed assumption of a drivingforce-independent mobility. It is this dependence on the driving force that gives rise to the complicated variation of the grain boundary velocity with system size evident in Figure 4, which we are now in a position to explain. At very small system sizes the formation of islands is frozen out and migration takes place via the simultaneous coordinated shuffling of all the atoms in the vicinity of the boundary. In these smallest systems an increase in the system size then implies that more atoms must be involved in the collective shuffle and so the velocity initially decreases with increasing system size. Only above some critical system size, which itself is dependent on the applied driving force, does it become possible to accommodate the island nuclei that are required in order to obtain a size-converged velocity.

In systems only just larger than the critical size only a single island will form and will grow to coalesce with itself across the periodic boundaries of the simulation cell. This single-island migration process can be seen in the smaller of the two systems illustrated in Figure 5 As the system size is further increased, the grain boundary area on which the single island can spontaneously form increases and so the velocity of the grain boundary increases. Only at relatively large system sizes can we accommodate the statistically converged population of critically stable islands (typically 2-3 islands) at the correct density and so measure a converged grain boundary velocity. The variation in the migration mechanism is illustrated schematically in the inset to Fig. 4.

We note that at higher driving forces, size-convergence can occur in relatively small simulation cells. The sizedependent effects that we observe only appear when sufficiently small driving forces are included in the analysis, otherwise, such effects may remain hidden.

Figure 9 presents a map of the mesoscale migration mechanism as a function of driving force and system size. The figure shows the value of $r_{\text {crit }}(P, T=600 \mathrm{~K})$ and also the minimum system size at which the grain boundary velocity is converged (based on the data in Fig. 4). We have also marked the range of system sizes and driving forces used in simulations of grain boundary migration in the literature. It is important to note that such simulations are unlikely to be system-size converged and that studies of mobility are often carried out in a region of parameter space in which the migration mechanism changes qualitatively.

We have shown above that the grain boundary velocity measured in MD simulations is only converged when large systems are used. To investigate the correct kinetics of flat boundaries, we have therefore carried out a series of grain boundary migration simulations across a range of temperatures and driving forces in large systems with grain boundary areas up to $425 \times 340 \AA^{2}$ (containing over a million atoms). An Arrhenius plot of the results of these simulations is shown in Figure 10, which provides a properly converged view of the behaviour of the grain boundary velocity with driving force and temperature. In contrast, the results shown in Figures 2 and 3 are derived from simulations carried out in a relatively small system of fixed size (typical of many similar studies in the literature) and incorporate the spurious effects of a varying migration mechanism. The form of these size-converged results is unexpectedly complex: the activation energy appears to vary with both driving force and temperature. Strongly non-Arrhenius behaviour, which can be seen in the strong deviations from linearity in Figure 10, is however consistent with the island-based migration mechanism as we show below.

From Eq. 7 we can derive expressions for the critical island radius and the free energy barrier for grain boundary migration:

$$
\begin{gathered}
r_{\text {crit }}(P, T)=\phi(T) / P d, \\
F_{\mathrm{A}}(P, T)=\pi[\phi(T)]^{2} / P d .
\end{gathered}
$$

We then assume the simplest possible model for the island edge entropy density, such that $\sigma \propto \gamma$ [49]. This model has a simple physical interpretation in terms of the grain boundary roughening transition as follows. Various studies have shown that above a given temperature, which depends on the boundary geometry, grain boundaries become "roughened" and exhibit thermal fluctuations in the shape of the grain boundary surface [12]. In terms of our model of the island free energy, the roughening transition corresponds to the temperature, call it $T_{\mathrm{c}}$, at which the free energy cost of forming island edge structures becomes zero, i.e. $\phi\left(T_{\mathrm{c}}\right)=0$. We thus assume,

$$
\sigma=\gamma / T_{\mathrm{c}},
$$

and obtain $T_{\mathrm{c}} \approx 700 \mathrm{~K}$ by studying the thickness [50] of an undriven grain boundary in an MD simulation as a function of temperature. We thus have,

$$
\phi(T)=\gamma\left(1-T / T_{\mathrm{c}}\right) .
$$


The process of grain boundary migration can be divided into two components: the nucleation of stable islands and the growth of those islands. The growth process involves repeated coordinated shuffles of groups of seven atoms. The barrier for these shuffles is relatively small $(\sim 0.1 \mathrm{eV})$ and so, except at very large driving forces, the rate limiting process will be that of nucleation, with the barrier $F_{\mathrm{A}}$.

Simple rate theory predicts a grain boundary velocity of $v=\lambda_{0} d \exp \left(-F_{\mathrm{A}} / k_{\mathrm{B}} T\right)$, where $\lambda_{0}$ is an attempt frequency and $d$ is the distance moved by the grain boundary in each migration process (i.e. the height of the island edge). Inserting our model for $F_{\mathrm{A}}$ and taking the logarithm gives a relationship of the form,

$$
\ln v=\ln v_{0}-\frac{U_{0}}{k_{\mathrm{B}} T}\left[1+\left(\frac{T}{T_{\mathrm{c}}}\right)^{2}\right] .
$$

Fitting the velocity and energy parameters $v_{0}$ and $U_{0}$ to the data from our size-converged migration simulations gives the lines shown in Figure 10. The accuracy of the fits confirms the correctness of the island-based model of migration kinetics. We can go further, because the island model also gives predictions for $v_{0}$ and $U_{0}$ :

$$
\begin{gathered}
\ln v_{0}=\ln \left(\lambda_{0} d\right)+2 \pi \gamma^{2} / k_{\mathrm{B}} T_{\mathrm{c}} P d, \\
U_{0}=\pi \gamma^{2} / P d \quad\left(=F_{\mathrm{A}}(P, T=0)\right),
\end{gathered}
$$

in which all the parameters are independently determined: $\gamma$ from the molecular statics calculations shown in Fig. 7 and $T_{\mathrm{c}}$ from MD simulations of the thickness of a stationary boundary. Figure 11(a) shows the predictions for $U_{0}$.

The first term in Eq. 13 is small compared to the second, being of order 1 whilst $\ln v_{0}$ has values between about 16 and 160 . The island model thus predicts that the ratio between the fitted energy term $U_{0}$ and logarithmic prefactor $\ln v_{0}$ will be (from Equations 13 and 14) $\ln v_{0} / U_{0}=2 / k_{\mathrm{B}} T_{\mathrm{c}}=33.1 \mathrm{eV}^{-1}$. Thus for an islandbased mesoscale migration mechanism a compensation effect with varying driving force arises quite naturally because both the internal energy and the entropy of a stable island nucleus vary as $\sim 1 / P$. In Figure 11 (b) the predicted compensation effect is compared with the data fitted to the size-converged migration simulations.

The quality of the fits in Figures 11(a) and (b) confirms the ability of the island-based mechanism to account quantitatively for the results of dynamical simulations of grain boundary migration.

A key feature of the island-based migration mechanism is that it implies that the energy barrier for grain boundary motion is inversely proportional to the driving force, as shown in Eq. 9 and illustrated in Fig. 12. Fig. 12 also shows the free energy barrier at an elevated temperature of $600 \mathrm{~K}$ at which the effect of the excess entropy of the island edge significantly reduces the barrier. As the driving force approaches experimentally realistic levels, the energy barrier explodes. Employing our model we find that at a realistic driving force of $10^{-2} \mathrm{MPa}\left(10^{-3} \mathrm{meV} /\right.$ atom $)$ the internal energy barrier will be several hundred eV with a critical island radius of several $\mu \mathrm{m}$. Only at temperatures within a few degrees of the roughening transition temperature will the effect of the entropy of the island edge reduce the free energy barrier for migration to a value that permits migration. Clearly under realistic conditions defect-free, flat grain boundaries are strictly immobile. This calls into question the concept of an intrinsic mobility for such boundaries. Such a mobility may exist at temperatures above the roughening transition, when the spontaneous thermal defects that fluctuate in and out of existence on the boundary at all finite temperatures can be formed at zero free energy cost (indeed, we see this behaviour in our results at $T=800 \mathrm{~K}$ in Figure 2). But any experimentally observed motion of grain boundaries below the roughening transition temperature must be a consequence of the presence of extended structural defects that remove the need for a homogeneous nucleation event. In this case, the mobility becomes a function of the morphology, or defect content, of the boundary and is no longer an intrinsic property of a boundary of a given geometry.

In Figure 2 we also observed a convergence to a fixed nominal mobility at very high driving force, even at temperatures well below the roughening temperature. Olmsted et al. 12] have explained this in terms of a "dynamical roughening" effect. However, this dynamical roughening is different in character from the normal thermal roughening: the former is a driven process, in which small island features are stabilised by very high driving forces, whereas in the latter case the free energy cost of the spontaneous formation of small islands becomes zero. The nominal mobility at high force is also greater than that at high temperature, at least in part because the thermal disorder in the thermally roughened boundary will hinder the ordered atomic shuffling by which the crystal volume transforms from one orientation to the other.

The island-based mechanism of grain boundary migration presented above differs qualitatively from models previously presented in the literature. Although Mott's originally proposed model [24] involved the transformation of "islands" of material, he assumed these islands to be of a fixed characteristic size (of around twenty atoms), leading to a fixed, i.e. driving-force-independent, energy barrier for grain boundary migration. An alternative mechanism was proposed by Gleiter [51], who considered atom-by-atom processes of grain boundary migration. Neither model is able to explain driving-forcedependent migration barriers.

The kinetics of thin film growth (or layer-by-layer) growth have been studied in great detail in the literature 52 54]. Whereas the driving forces for thin film growth can be very large (a few tenths of an $\mathrm{eV}$ per atom), the realistic limit for grain boundary migration is the zero- 
force limit $\left(10^{-9}-10^{-4} \mathrm{eV} /\right.$ atom $)$. At realistic driving forces, grain boundary migration is therefore very firmly in the nucleation-limited modes discussed by Kashchiev 52 and Avrami [53, 54]. Indeed, even at the highest of the driving forces that we use in our simulations, the energy needed to form a critical island nucleus is orders of magnitude larger than the barrier for its incremental growth.

Kang et al. have recently suggested that the cost of forming island nuclei would account for their observations of a critical minimum driving force for grain boundary migration in $\mathrm{BaTiO}_{3}$ 43. They suggest that the same principles that are used to model crystal growth in two phase systems could profitably be applied to single phase solid systems. Our findings and analytical model nicely explain these experimental results.

Deng and Schuh 8] have presented a modified version of the interface random walk method 16] for determining grain boundary mobility even at very low driving forces and temperatures. Using this approach (the AIRwalk method) they have identified two regimes of grain boundary migration [9]: a "diffusional" regime at low driving forces and a "ballistic" regime at high driving forces (and high temperatures). 9] We also observe ballistic migration of our boundary at very high driving forces, but the behaviour we discuss here is outside this ballistic regime. Whereas Deng and Schuh [9] are able to explain a nonlinear relation between driving force and velocity in terms of a transition between two different regimes of migration, the non-linearity that we observe is not related to such a transition. The non-linearity here is a direct result of the island-based mechanism: as the driving force decreases, the free energy barrier for migration increases inversely, because the radius of a stable island nucleus increases.

Using the AIRwalk method [8], Deng and Schuh are also able to detect residual mobility down to very low driving forces [9]. This implies that an intrinsic mobility does exist. In contrast, the island-based mechanism implies that mobility at zero driving force is strictly zero (in the sense that $\lim _{P \rightarrow 0} \mathrm{~d} V / \mathrm{d} P=0$ ). These different conclusions are, we believe, a result of the nature of the nuclei which must form spontaneously in order to effect grain boundary motion. In a thin-slab system, as studied in Ref. 9], the island nuclei (effectively disconnection loops) in our systems become a pair of short line defects (disconnections). The implications for the kinetics of migration are likely to be significant.

The role of steps (or disconnections) in grain boundary migration in a small system has previously been reported in the context of shear-coupled migration and is extensively discussed by Rajabzadeh et al. [29]. In Ref. 29] the formation of pairs of short disconnections is driven by the applied shear. In a larger system the boundary migration would likely require the nucleation of disconnection loops, bounding islands similar to those that we find. The interaction of these disconnection loops with the applied shear would be complex.
Mendelev et al. 55] have studied the migration of a $\Sigma 5$ asymmetric tilt boundary in which the elastic driving force of Zhang et al. 17] and the modified random walk method of Deng and Schuh [8] are directly compared. Both approaches yield the same mobility and the absence of a driving force in the random walk method suggests that what is being measured is indeed an intrinsic mobility. Importantly, Ref. [55] makes use of a fully threedimensional and reasonably large system $(50 \AA \times 50 \AA$ in the grain boundary plane). In contrast, the symmetric $\Sigma 7$ boundary that we study here shows no intrinsic mobility i.e. this boundary is immobile in the zero-drivingforce limit. This apparent contradiction is related to the nature of the boundaries simulated. In Ref. [55] an asymmetric boundary is used, which contains structural features perpendicular to the boundary plane larger than the atomic-scale ridges in the symmetric $\Sigma 7$ boundary that we simulate. These features act as nucleation sites for the formation of the defects required for migration and so remove the need for a homogeneous nucleation process (in the symmetric $\Sigma 7$ boundary, no preferential sites for nucleation exist). Song and Hoyt [56, 57] have similarly found that heterogenous nucleation is active in simulations of the migration of interphase boundaries in iron in the case of certain boundaries. We have found that grain boundary morphology and structural defect content can dramatically affect migration kinetics and a future paper will address this issue in detail.

Here we have focused our analysis on simulations of a single low- $\Sigma$ boundary, typical of those used in computational studies. We have, however, also simulated a much longer period $\Sigma 9183$ tilt boundary close to the $\Sigma 7$ boundary discussed here (the misorientation angle is $40.17^{\circ}$ as opposed to $38.21^{\circ}$ ). For this boundary we find the same type of island-based migration process and the same complex dependence of velocity on driving force and temperature. In the case of more general boundaries, the edge of the island structures will not be a pure step, but will have some dislocation content with long range strain fields. Whilst these strain fields will add a component to the energy of an island nucleus dependent on its radius, the relevant limit is the low driving force one. At low force, the critical islands will be very large and the strain component in the edge energy will likely be negligible.

\section{CONCLUSIONS}

We have presented the results of dynamical simulations of the migration of a typical low- $\Sigma$ symmetric tilt boundary across broad ranges of driving forces and system sizes. To ensure that our boundary migration simulations were properly converged we employed larger simulations cells and smaller driving forces than in typical studies in the literature, i.e. we have moved systematically towards the experimental limit of very large system size and very low driving force. The grain boundary velocities obtained in our simulations violated several of the key assump- 
tions concerning grain boundary kinetics: the velocity was not generally proportional to the driving force for grain boundary motion, the energy barrier for migration varied strongly with driving force and with temperature, and the kinetics of migration showed a strong and complicated dependence on the size of the system being simulated.

Since a converged description of grain boundary migration was only achieved at large system sizes, we carried out a detailed analysis of the migration mechanism at this length-scale, i.e. the mesoscale. By examining the evolving shape of migrating grain boundaries in dynamical simulations in very large systems we were able to identify the rate-limiting process: an island-based migration mechanism. Flat grain boundaries move by the nucleation, growth, and coalescence of islands of transformed material on the grain boundary surface. We developed an analytical model for this island-based migration mechanism and derived the key parameters for the model from calculations of static grain boundaries, independent of the dynamical simulations. We showed that the analytical island model accurately reproduces the complex variation of the grain boundary velocity with driving force and temperature. We identified several different modes of grain boundary migration, dependent on driving force and system size. In artificially small systems the islandbased mechanism is frozen out and the grain boundary migrates via a simultaneous rearrangement of all grain boundary atoms, a process with a lower energy barrier. In larger systems a single island can form, coalescing with itself across the periodic boundaries of the simulation cell. Only in fully converged large systems can a multiple-island-based migration mechanism become operational. The transition between the different modes of migration explains the complex system size dependence of the grain boundary velocity. Due to computational constraints, studies in the literature typically make use of systems that are substantially smaller than those that we find to be necessary for size-converged migration results.

The migration of defect-free, flat grain boundaries via the formation and growth of islands involves the kinetics of homogeneous nucleation. Our analytical model for this process highlights that the energy barrier for grain boundary migration is inversely proportional to the driving force. This very strong driving force dependence is verified by the results of our dynamical simulations in large systems at low driving force. The implication is that in the range of experimental driving forces defectfree, flat grain boundaries become immobile, implying that the concept of an intrinsic mobility becomes obsolete for such boundaries. When such boundaries are observed to move in experiment this is, we suggest, a consequence of being above the roughening transition temperature or of the presence of structural defects in the boundary. In the latter case the mobility will be a property of the defect content, rather than an intrinsic property of the boundary associated with its geometry.

We have highlighted the important role of the mesoscale in determining mechanisms of grain boundary migration and how these mechanisms qualitatively modify the migration kinetics, i.e. the dependence of migration velocity on driving force and temperature. For low driving forces and simulation cells sufficiently large to accommodate the mesoscale features a migration mechanism based on island nucleation has been uncovered. This mechanism shows a fundamentally different asymptotic behaviour at zero driving force to previously discussed mechanisms. It will be interesting to see if even lower driving forces are associated with further mesoscale mechanisms, which may have different asymptotic behaviour. The critically important and qualitative implications of size convergence and dimensionality for deriving grain boundary mobility data from MD simulations, discovered and analysed in this study, should be accounted for in future work and are likely to further impact our understanding of grain boundary kinetics.

\section{ACKNOWLEDGMENTS}

C.P.R. was funded by a Postdoctoral Fellowship of the Alexander von Humboldt Foundation.

\section{REFERENCES}

[1] C. H. Li, E. H. Edwards, J. Washburn, and E. R. Parker, Acta Metallurgica 1, 223 (1953).

[2] G. Gottstein and L. S. Shvindlerman, Scripta Metallurgica et Materialia 27, 1515 (1992).

[3] Y. Huang and F. J. Humphreys, Acta Materialia 47, 2259 (1999).

[4] M. Winning, G. Gottstein, and L. S. Shvindlerman, Acta Materialia 50, 353 (2002).

[5] G. Gottstein, D. A. Molodov, L. S. Shvindlerman, D. J. Srolovitz, and M. Winning, Current Opinion in Solid State and Materials Science 5, 9 (2001).
[6] M. Winning, Philosophical Magazine 87, 5017 (2007).

[7] D. A. Molodov, U. Czubayko, G. Gottstein, and L. S. Shvindlerman, Acta Materialia 46, 553 (1998).

[8] C. Deng and C. Schuh, Physical Review Letters 106, 45503 (2011).

[9] C. Deng and C. A. Schuh, Physical Review B 84, 214102 (2011).

[10] K. G. F. Janssens, D. Olmsted, E. A. Holm, S. M. Foiles, S. J. Plimpton, and P. M. Derlet, Nat Mater 5, 124 (2006).

[11] R.-J. Jhan and P. D. Bristowe, Scripta Metallurgica et 
Materialia 24, 1313 (1990).

[12] D. L. Olmsted, S. M. Foiles, and E. A. Holm, Scripta Materialia 57, 1161 (2007).

[13] D. L. Olmsted, E. A. Holm, and S. M. Foiles, Acta Materialia 57, 3704 (2009).

[14] B. Schonfelder, G. Gottstein, and L. Shvindlerman, Metallurgical and Materials Transactions A 37, 1757 (June 2006).

[15] B. Schönfelder, D. Wolf, S. R. Phillpot, and M. Furtkamp, Interface Science 5, 245 (1997).

[16] Z. T. Trautt, M. Upmanyu, and A. Karma, Science 314, 632 (2006).

[17] H. Zhang, M. I. Mendelev, and D. J. Srolovitz, Acta Materialia 52, 2569 (2004).

[18] H. Zhang, D. J. Srolovitz, J. F. Douglas, and J. A. Warren, Acta Materialia 55, 4527 (2007).

[19] H. Zhang, M. I. Mendelev, and D. J. Srolovitz, Scripta Materialia 52, 1193 (2005).

[20] H. Zhang and D. J. Srolovitz, Acta Materialia 54, 623 (2006).

[21] J. Zhou and V. Mohles, Acta Materialia 59, 5997 (2011).

[22] G. Gottstein and L. Shvindlerman, Grain Boundary Migration in Metals - Thermodynamics, Kinetics, Applications (CRC Press, 2010).

[23] A. P. Sutton and R. W. Balluffi, Interfaces in crystalline materials (Oxford: Clarendon Press, 1995).

[24] N. F. Mott, Proceedings of the Physical Society 60, 391 (1948).

[25] R. Smoluchowski, Physical Review 83, 69 (1951).

[26] K. L. Merkle and L. J. Thompson, Materials Letters 48, 188 (2001).

[27] R. Würschum and R. W. Balluffi, physica status solidi (a) 136, 323 (1993).

[28] L. Zhou, N. Zhou, and G. Song, Philosophical Magazine 86, 5885 (2006)

[29] A. Rajabzadeh, F. Mompiou, M. Legros, and N. Combe, Physical Review Letters 110, 265507 (2013).

[30] In the high driving force limit, the velocity would then be limited by the attempt frequency: $\lim _{\varepsilon \rightarrow \infty} v(\varepsilon, T)=$ $\left(\lim _{\varepsilon \rightarrow \infty} f(\varepsilon)\right) \exp \left(-E_{\mathrm{A}} / k_{\mathrm{B}} T\right)$.

[31] One recent paper [21] implicitly assumes that the reverse barrier is unaffected by the driving force such that $e=0$. In the low driving force limit, this assumption gives the same linear dependence of $v$ on $\varepsilon$. At high driving force, however, the velocity increases exponentially, without limit.

[32] S. Plimpton, Journal of Computational Physics 117, 1 (1995).

[33] Our largest cells have a boundary area density of $\sim$
$0.005 \AA^{-1}$, equivalent to a grain size of $\sim 100 \mathrm{~nm}$ across.

[34] J. W. Cahn, Y. Mishin, and A. Suzuki, Acta Materialia 54, 4953 (2006).

[35] A. Karma, Z. T. Trautt, and Y. Mishin, Physical Review Letters 109, 095501 (2012)

[36] V. A. Ivanov and Y. Mishin, Physical Review B 78 (2008)

[37] E. R. Homer, S. M. Foiles, E. A. Holm, and D. L. Olmsted, Acta Materialia 61, 1048 (2013).

[38] H. A. Khater, A. Serra, R. C. Pond, and J. P. Hirth, Acta Materialia 60, 2007 (2012)

[39] F. Mompiou, D. Caillard, and M. Legros, Acta Materialia 57, 2198 (2009)

[40] A. Rajabzadeh, M. Legros, N. Combe, F. Mompiou, and D. A. Molodov, Philosophical Magazine, Philosophical Magazine 93, 1299 (2013)

[41] R. R. Zope and Y. Mishin, Phys. Rev. B 68, 024102 (2003).

[42] C. P. Race, To be Published.

[43] S.-J. L. Kang, M.-G. Lee, and S.-M. An, Journal of the American Ceramic Society 92, 1464 (2009).

[44] Because thermal fluctuations shift the effective driving force away from the nominal value, we interpolate $v$ as a function of the effective driving force to make our Arrhenius plots. Any errors introduced by this procedure are insignificant compared with variations due to the statistical nature of the migration process.

[45] B. E. C. Banks, V. Damjanovic, and C. A. Vernon, Nature 240, 147 (1972).

[46] H. Zhang, D. J. Srolovitz, J. F. Douglas, and J. A. Warren, Physical Review B $\mathbf{7 4}$ (2006).

[47] J. A. Venables, G. D. T. Spiller, and M. Hanbucken, Reports on Progress in Physics 47, 399 (1984).

[48] H. Zhang, M. Upmanyu, and D. Srolovitz, Acta Materialia 53, 79 (2005).

[49] T. Zhu, J. Li, A. Samanta, A. Leach, and K. Gall, Physical Review Letters 100, 025502 (2008).

[50] In this case identified as the distance normal to the grain boundary plane over which the order parameter associated with the artificial driving force varies from its maximum to minimum values.

[51] H. Gleiter, Acta Metallurgica 17, 565 (1969).

[52] D. Kashchiev, Journal of Crystal Growth 40, 29 (1977)

[53] M. Avrami, The Journal of Chemical Physics 8, 212 (2004)

[54] M. Avrami, The Journal of Chemical Physics 7, 1103 (2004)

[55] M. I. Mendelev, C. Deng, C. A.

Schuh, and D. J. Srolovitz, Modelling and Simulation in Materials Science and Engineering 21,

[56] H. Song and J. J. Hoyt, Acta Materialia 61, 1189 (2013).

[57] H. Song and J. J. Hoyt, Acta Materialia 60, 4328 (2012) 


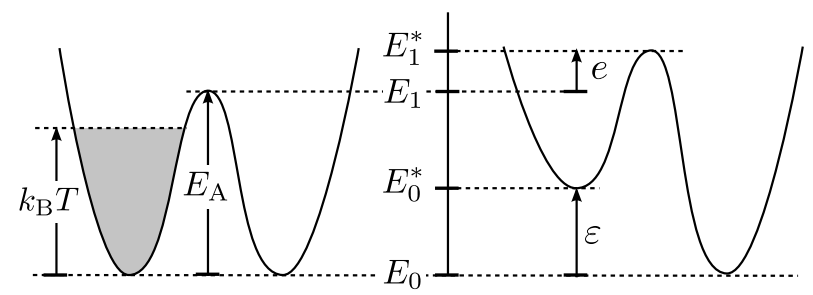

FIG. 1. A schematic illustration of a simple barrier hopping model of grain boundary migration. The labelled energies are discussed in detail in Section $\amalg \mathrm{A}$ 


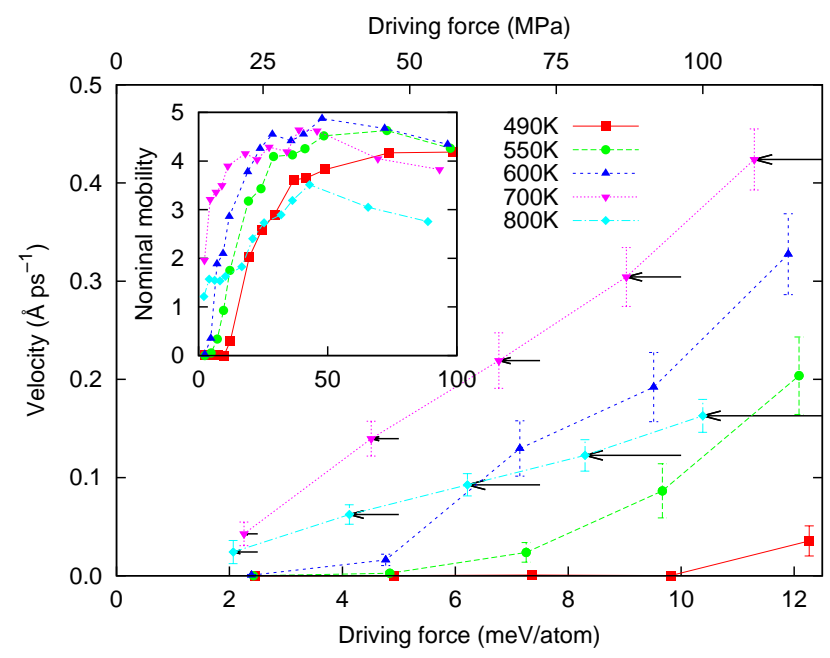

FIG. 2. Results of grain boundary migration simulations showing velocity as a function of driving force over a range of temperatures. The error bars show an estimate of one standard deviation in the measured velocity (derived using bootstrap resampling [42]). The horizontal arrows indicate the adjustments to the nominal driving force strength required to account for thermal fluctuations. The inset shows the nominal mobility (see main text for a definition) as a function of driving force (in $\mathrm{eV} /$ atom) up to very high driving forces.

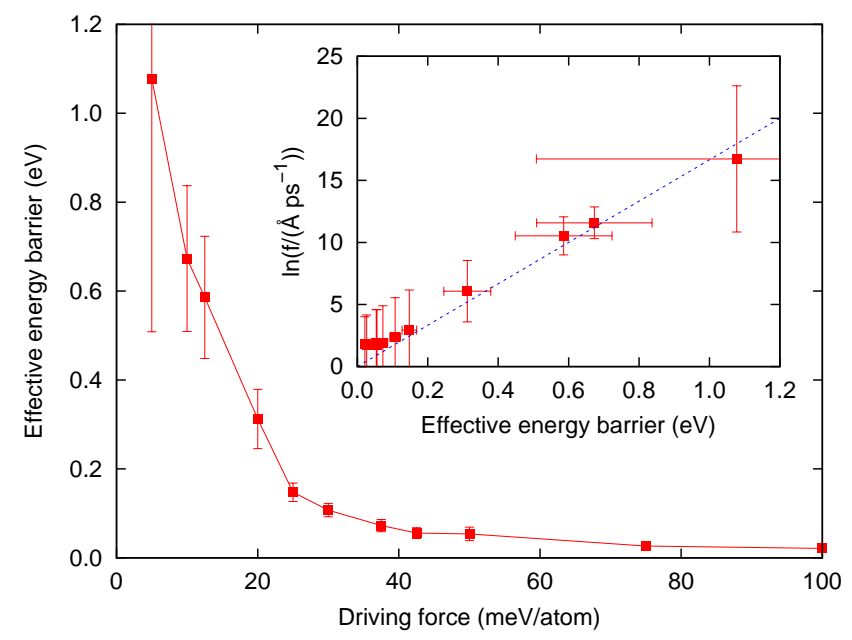

FIG. 3. Results derived from fitting to Arrhenius plots of the data in Fig. 2 as a function of driving force. Main figure: activation energy - the solid (red) line is a guide for the eye. Inset: the compensation effect - the dashed (blue) line is a linear fit to the data as a guide for the eye. 


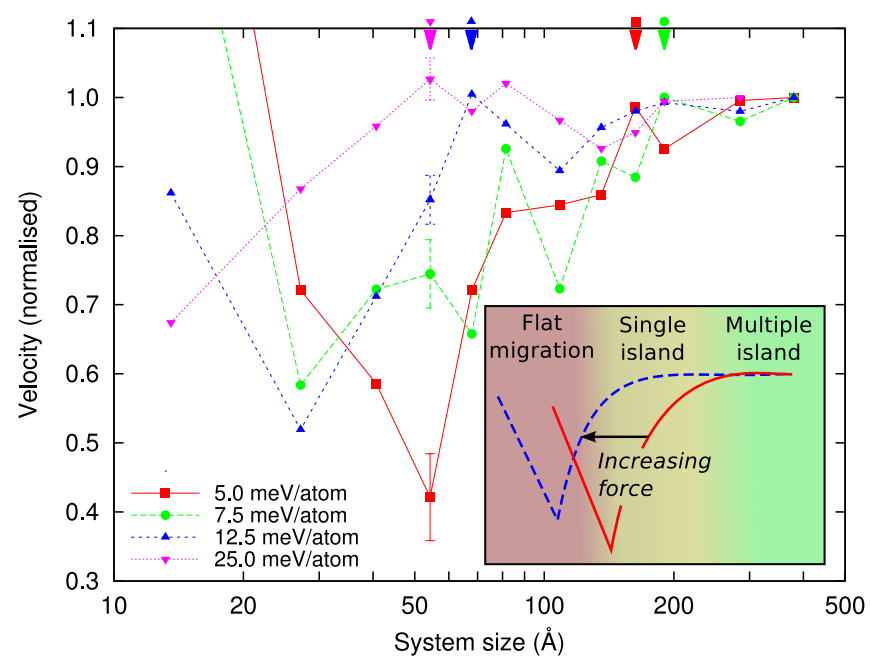

FIG. 4. The velocity of a grain boundary in MD simulations at $600 \mathrm{~K}$ as a function of system size (length of simulation cell in the dimensions in the grain boundary plane) and driving force. The velocities are normalised to the value in the largest system at each value of the driving force. Representative error bars (one standard deviation in the estimated velocity 42]) are shown for a single system size. The coloured arrows mark the approximate minimum system size at which convergence in the velocity occurs. Corresponding points are marked in Fig. 9. The inset shows a schematic of the observed trends and is discussed in Section IIIB
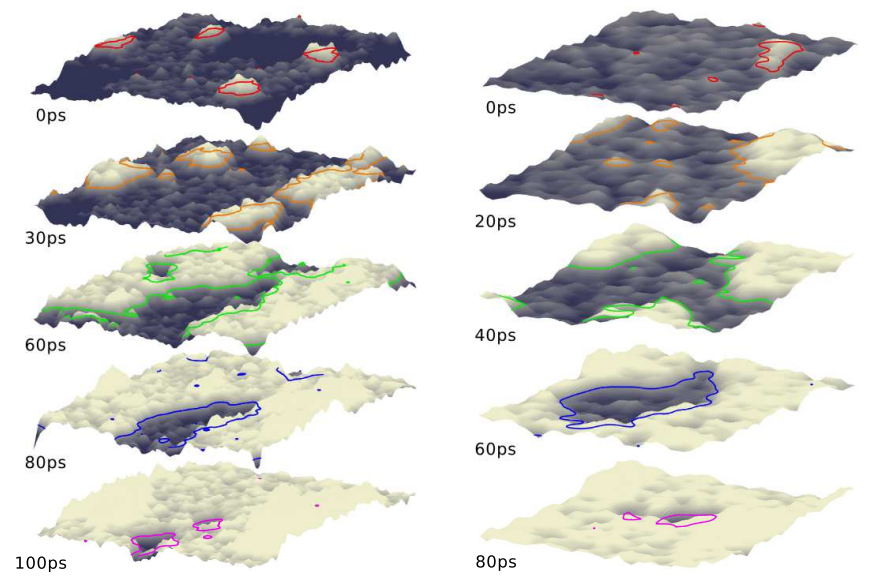

FIG. 5. Snapshots of a migrating grain boundary surface. The contours show the edges of (white) islands of migrated grain boundary area at different times for a large system $(340 \times$ $420 \AA^{2}$, left $)$ and a smaller system $\left(170 \times 210 \AA^{2} \approx 4 r_{\text {crit }}\right.$, where $r_{\text {crit }}$ is the radius of a critically stable island nucleus), both with a driving force of $5 \mathrm{meV} /$ atom at $600 \mathrm{~K}$. 

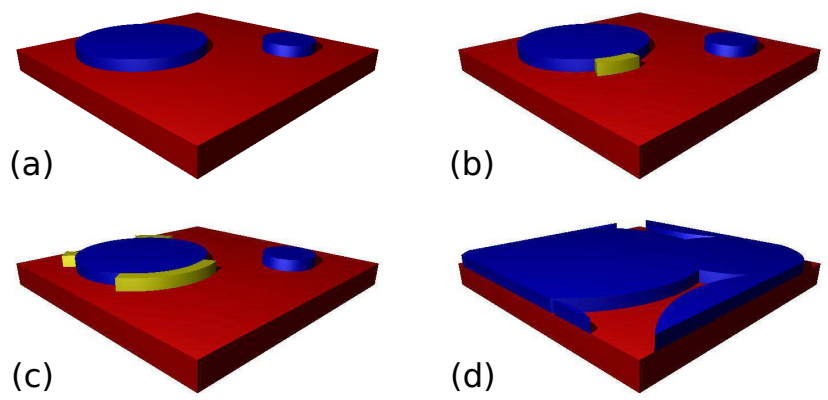

FIG. 6. A schematic illustration of the island nucleation and growth model of grain boundary migration. The solid volume represents crystal volume in the favoured orientation. The upper half of the bicrystal, in the disfavoured orientation, is not shown. (a) Islands (blue) form on the surface of the flat boundary (red) due to thermal fluctuations. (b) Double kinks (yellow) form in the island edges and (c) migrate and coalesce to give growth of the islands. (d) The islands grow until they coalesce, at which point the grain boundary has migrated by the edge height.

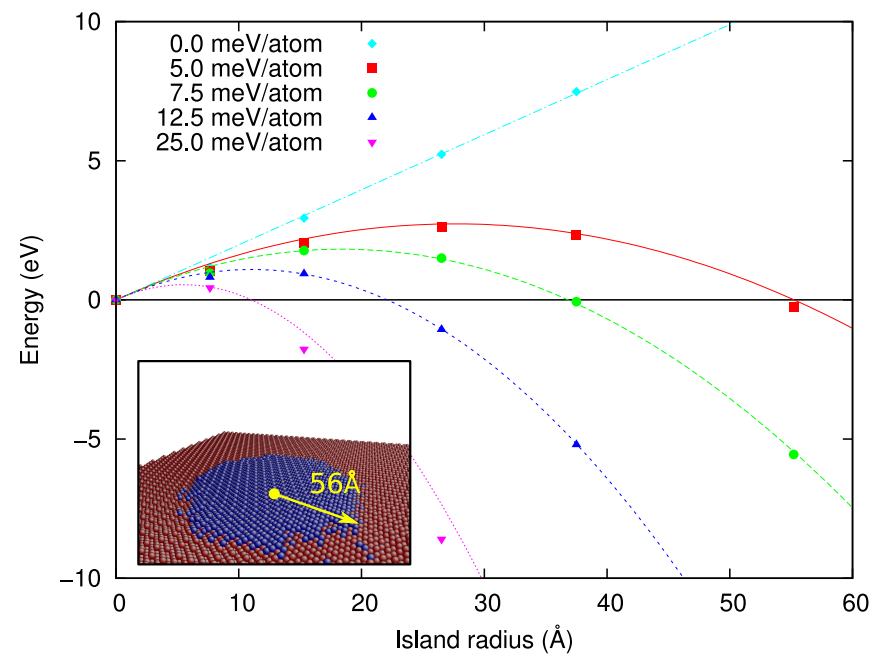

FIG. 7. The excess energies of circular islands in a grain boundary surface. The symbols show the results of static atomistic calculations (see inset) using an EAM potential. The curves are predictions of the analytical model in Eq. 7 (see main text). 


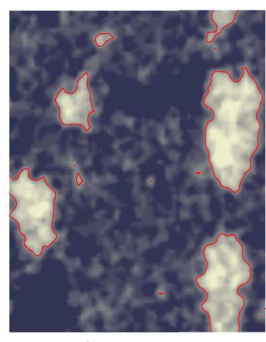

$5.0 \mathrm{meV} / \mathrm{atom}$

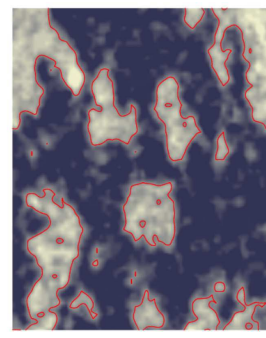

$7.5 \mathrm{meV} / \mathrm{atom}$

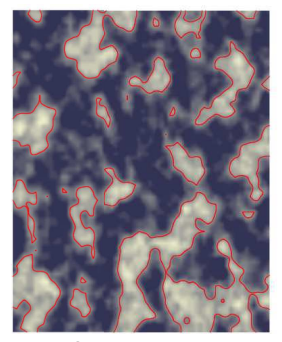

$12.5 \mathrm{meV} / \mathrm{atom}$

FIG. 8. Snapshots of islands of migrated grain boundary area at an early stage in the migration process (approximately the critically stable island population) as a function of driving force in a system $340 \AA \times 420 \AA$.

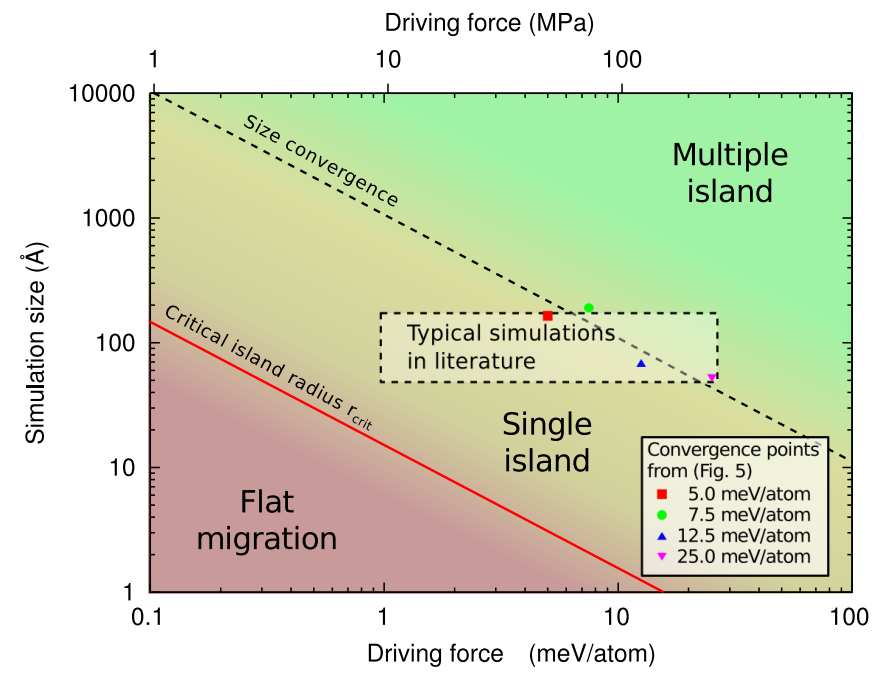

FIG. 9. A map of the grain boundary migration mechanism as a function of driving force and system size. The solid red line shows the critical radius $r_{\text {crit }}$ from the analytical model in Eq. 7] Typical parameters [10, 13, 14, 48] of simulations in the literature are marked. The symbols (and dashed line) mark the threshold for size convergence of the grain boundary velocity from Figure 4 


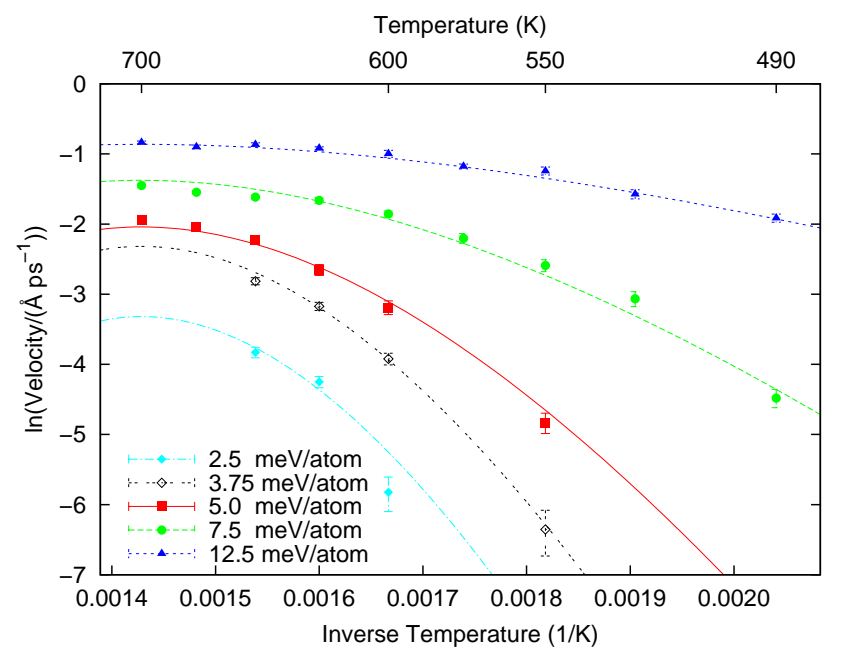

FIG. 10. Arrhenius plots of the size-converged velocity of grain boundaries. The lines show the results of fitting the analytical model of island-based kinetics in Eq. 12 as discussed in the text. Note that the roughening transition temperature is $\sim 700 \mathrm{~K}$.
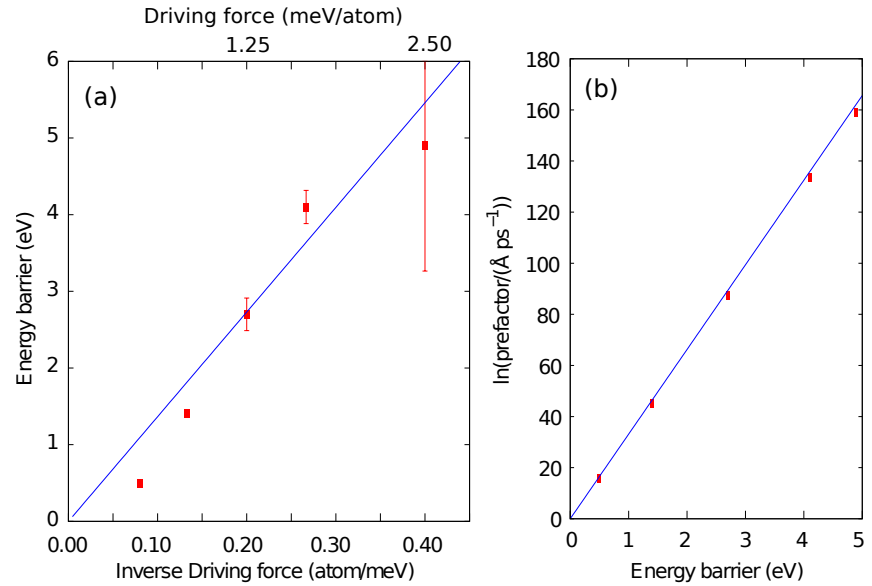

FIG. 11. (a) A comparison of (points) the internal energy barriers extracted from dynamical simulations and (line) the analytical predictions of Eq. 14 (b) The compensation effect (a proportionality between the logarithm of the kinetic pre-factor and the internal energy barrier) arises as a natural consequence of the island-based migration mechanism. The points are extracted from dynamical simulations and the line is the prediction of the analytical model. 


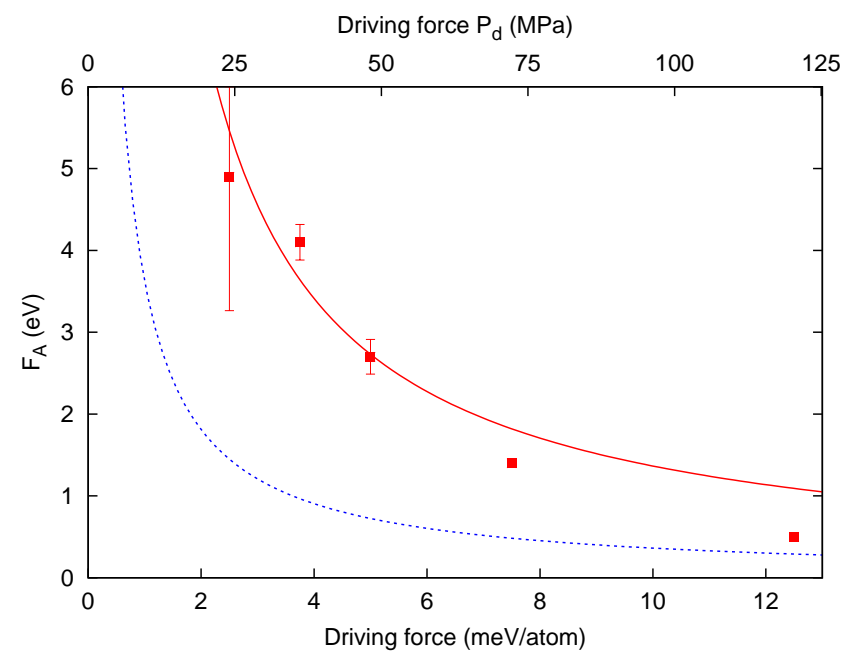

FIG. 12. The energy barrier for grain boundary migration as a function of driving force. The points are the internal energy barrier $U_{0}$ (equivalently $F_{\mathrm{A}}(P, T=0$ ) extracted from the dynamical simulations by fitting the data in Figure 10. The solid (red) line is the prediction of Eq. 14 (or of Eq. 9 at $T=0 \mathrm{~K}$ ). The dashed (blue) line is the free energy barrier $F_{\mathrm{A}}$ at $600 \mathrm{~K}$ from Eq. 9 